\title{
OXIFERTIRRIGACIÓN QUÍMICA MEDIANTE RIEGO EN TOMATE HIDROPÓNICO CULTIVADO EN INVERNADERO ${ }^{1}$
}

\author{
Freddy Soto-Bravo ${ }^{2}$
}

\begin{abstract}
RESUMEN
Oxifertirrigación química mediante riego en tomate hidropónico cultivado en invernadero. El objetivo del presente trabajo fue evaluar el efecto del peróxido de hidrógeno $\left(\mathrm{H}_{2} \mathrm{O}_{2}\right)$ como fuente de oxígeno en la rizosfera, en tomate injertado (cv. Durinta/cv. Maxifor) y utilizando fibra de coco como sustrato. El estudio se llevó a cabo entre los años 2009 y 2010. Se utilizaron dos tratamientos: un control $\sin \mathrm{H}_{2} \mathrm{O}_{2}\left(\mathrm{~T}_{0}\right)$ y otro con $\mathrm{H}_{2} \mathrm{O}_{2}\left(\mathrm{~T}_{1}\right)$ aplicado en cada riego. Se evaluaron parámetros de: $i$ - fertirrigación: oxígeno $\left(\mathrm{O}_{2}\right)$, $\mathrm{pH}$, conductividad eléctrica (CE) y porcentaje de drenaje; $i i$ crecimiento: diámetro basal y altura de planta; $i i i$ - rendimiento y $i v$ - calidad de fruto: firmeza, grados Brix, peso seco y $\mathrm{pH}$. Durante el ciclo de vida del cultivo, el valor promedio de $\left[\mathrm{O}_{2}\right]$ en la solución de riego fue $9,92 \mathrm{mg} / 1$ en $\mathrm{T}_{0}$ y $12,1 \mathrm{mg} / \mathrm{l}$ en $\mathrm{T}_{1}$, mientras que en la solución drenada la $\left[\mathrm{O}_{2}\right]$ fue de $8,75 \mathrm{mg} / \mathrm{l}$ en $\mathrm{T}_{0} \mathrm{y}$ de $9,22 \mathrm{mg} / \mathrm{l}$ en $\mathrm{T}_{1}$. Aunque se presentaron diferencias significativas $(\mathrm{P}<0,05)$ en $\left[\mathrm{O}_{2}\right]$ entre tratamientos durante algunos periodos del ciclo de cultivo, en el $\mathrm{T}_{0}$ la $\left[\mathrm{O}_{2}\right]$ no alcanzó un valor crítico que afectara la adecuada oxigenación de las raíces. Por tanto, no hubo efecto del tratamiento con peróxido de hidrógeno $\left(\mathrm{T}_{1}\right)$ sobre parámetros de fertirrigación, crecimiento, rendimiento y calidad del fruto del cultivo.
\end{abstract}

Palabras clave: química verde, rizosfera, hipoxia.

\begin{abstract}
Chemical oxifertigation through the irrigation of greenhouse hydroponic tomato crop. The aim of this study was to evaluate the effect of hydrogen peroxide $\left(\mathrm{H}_{2} \mathrm{O}_{2}\right)$ as an oxygen source in the rhizosphere, in grafted tomato (cv. Durinta/cv Maxifor) and using coconut fiber as substrate The study was conducted from 2009 to 2010. Two treatments were used: a control without $\left(\mathrm{H}_{2} \mathrm{O}_{2}\right)\left(\mathrm{T}_{0}\right)$ and the other with $\mathrm{H}_{2} \mathrm{O}_{2}\left(\mathrm{~T}_{1}\right)$ applied in each irrigation. The parameters evaluated were $i$ - fertigation: oxygen concentration $\left(\left[\mathrm{O}_{2}\right]\right)$, $\mathrm{pH}$, electrical conductivity (EC), and drainage percentage; ii- growth: basal diameter and plant height; iii- yield and $i v$ - fruit quality: firmness, Brix degrees, dry weight, and $\mathrm{pH}$. The average value of $\left[\mathrm{O}_{2}\right]$ in the irrigation solution through out the crop cycle increased from $9,92 \mathrm{mg} / \mathrm{l}$ at $\mathrm{T}_{0}$ to 12,1 $\mathrm{mg} / \mathrm{l}$ at $\mathrm{T}_{1}(\mathrm{P}<0,05)$, meanwhile in the drained solution the value increased from $8,75 \mathrm{mg} / \mathrm{l}$ at $\mathrm{T}_{0}$ to $9,22 \mathrm{mg} / \mathrm{l}$ at $\mathrm{T}_{1}$ $(\mathrm{P}<0,05)$. Although significant differences $(\mathrm{P}<0.05)$ were reached in the $\left[\mathrm{O}_{2}\right]$ between treatments during some periods of the crop cycle, the $\left[\mathrm{O}_{2}\right]$ in the $\mathrm{T}_{0}$ did not reach a critical threshold that would affect the proper oxygenation of the roots. Therefore, there was no effect of hydrogen peroxide treatment on the growth, productivity and quality of the fruit.
\end{abstract}

Keywords: green chemistry, rhizosphere, hypoxia.

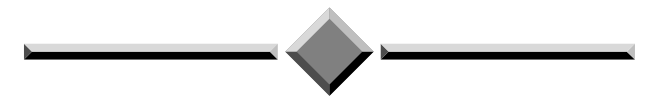

1 Recibido: 18 de junio, 2014. Aceptado: 28 de octubre, 2014. Parte de la tesis para obtener el grado de Máster Itinerario de Investigación Código 70313212 del Máster Oficial en Producción Vegetal en Cultivos Protegidos, Universidad de Almería, España.

2 Universidad de Costa Rica, Facultad de Ciencias Agroalimentarias, Estación Experimental Agrícola Fabio Baudrit Moreno. Alajuela, Costa Rica. Apdo postal: 183-4050 Alajuela, Costa Rica. freddy.sotobravo@ucr.ac.cr 


\section{INTRODUCCIÓN}

El cultivo hidropónico se caracteriza por poseer un sistema radical muy denso, confinado en un pequeño volumen de sustrato, con elevadas tasas de actividad metabólica, respiración y crecimiento. Como consecuencia, los requerimientos de $\mathrm{O}_{2}$ en la rizosfera incrementan sustancialmente (Urrestarazu, 2004). Algunos factores que afectan la $\left[\mathrm{O}_{2}\right]$ en la rizosfera son: capacidad de aireación del sustrato (Ansorena, 1994; Abad et al., 2004), volumen del sustrato por planta (Zhi et al., 2007), edad del sustrato (García et al., 1997), manejo del riego (Bonachela, et al., 2005), concentración de oxígeno en el agua de riego (Pívot et al., 1998), contenido de materia orgánica (Abad et al., 1997), temperatura ambiental (Bonachela et al., 2007), salinidad del sustrato (Barret-Lennard, 2003) y la forma y profundidad del contenedor (Urrestarazu, 2004).

Condiciones de hipoxia en el medio de cultivo afectan el crecimiento, el rendimiento de la planta y la calidad del producto de los cultivos hortícolas, debido a que se alteran procesos fisiológicos y bioquímicos tales como el potencial hídrico (Drew, 1997), el contenido de clorofila, fotosíntesis y balance hormonal por exceso de etileno (Irving et al., 2007; Horchani et al., 2008b, 2009). Además, se reduce la conductancia estomática (Vartapetian y Jackson, 1997), se afecta la absorción, el transporte de agua y nutrientes (Stiepniewski y Przywara, 1992), y es causa de muerte celular por acidificación citoplasmática (Drew, 1997) y epinastia foliar (Pezeshki, 2001).

La hipoxia produce oxidación y muerte de raíces (Horchani et al., 2008b) al disminuir la tasa fotosintética y el transporte de asimilados a las raíces; además del agotamiento progresivo de las reservas por el catabolismo de carbohidratos y proteínas (Devaux et al., 2003). La falta de oxígeno induce a la respiración anaeróbica, incrementando la concentración de etanol y $\mathrm{CO}_{2}$ en la rizosfera hasta niveles tóxicos (Irving et al., 2007). Los daños a nivel de tejidos y células incrementaron la susceptibilidad de las raíces a Phytium sp. en tomate (Solanum lycopersicum L.) hidropónico (Chérif et al., 1997).

En cultivos sin suelo se pueden presentar algunas condiciones que favorecen la hipoxia, tales como el aumento de la temperatura y la salinidad (Marfa y Gurí, 1999), una elevada densidad radicular y baja $\left[\mathrm{O}_{2}\right]$ en el agua de riego (Castellanos, 2006). La deficiencia de oxígeno suele ocurrir en épocas cálidas, debido al incremento de la temperatura del sustrato y consecuentemente de la tasa de respiración de las raíces (Bonachela, et al., 2005).

Considerando que la solución de riego es el vehículo para el aporte de oxígeno a las raíces, se han propuesto diferentes métodos de oxigenación en cultivos en sustrato. La oxifertirrigación pura consiste en la inyección de oxígeno gaseoso a presión en el agua de riego (Bhattarai et al., 2005; Marfa et al., 2005). En la oxifertirrigación física o mecánica se aplica agitación mecánica o inyección de aire directamente en el tanque de solución nutritiva (Castellanos, 2006; Alcayde, 2009). El método de oxifertirrigación química consiste en la aplicación de compuestos a base de peróxidos, las cuales durante el proceso de descomposición liberan oxígeno (Marfa y Gurí, 1999; Urrestarazu y Mazuela, 2005).

Bajo condiciones de hipoxia, la oxifertirrigación asegura un óptimo funcionamiento de la raíz, y mejora la actividad microbiana, las transformaciones minerales y la eficiencia de uso del agua (Bhattarai et al., 2005). Además, compensa los efectos negativos de la compactación y la salinidad sobre la aireación del medio de cultivo, mejorando el crecimiento y el rendimiento de las plantas. En tomate, Horchani et al. (2008a) observaron que bajo condiciones de hipoxia, la caída de flores y frutos debido a la acumulación de etileno, disminuyó la producción; sin embargo, no afectó el calibre y peso del fruto ya existente.

En diversos estudios realizados en el cultivo de tomate, los valores de $\left[\mathrm{O}_{2}\right]$ bajo los cuales se afecta el crecimiento, la producción y calidad de frutos, han sido variables, y se encuentran en un rango de 3 a 7 $\mathrm{mg} / \mathrm{l}$. Valores mínimos de $\left[\mathrm{O}_{2}\right]$ de $3 \mathrm{mg} / \mathrm{l}$ (Gislerod y Kempton, 1983), 5,3 mg/l(Zheng et al., 2007), 6,7 mg/l (Gislerod y Adam, 1983) y 65\% de saturación de $\mathrm{O}_{2}$ no afectaron el crecimiento y la producción. En cultivo de tomate en fibra de coco (Cocus nucífera), Castellanos (2006) no obtuvo efectos sobre la producción a partir de una $\left[\mathrm{O}_{2}\right]$ de $5,5 \mathrm{mg} / \mathrm{l}$.

Se han determinado umbrales de $\left[\mathrm{O}_{2}\right]$ con valores de $2,7 \mathrm{mg} / \mathrm{l}$ en pepino (Cucumis sativus) (Gislerod y Adam, 1983), 2,1 mg/l en lechuga (Lactuca sativa) (Goto et al., 1996) y $2 \mathrm{mg} / \mathrm{l}$ en pimiento (Capsicum аппиит) (Ehret et al., 2010), por debajo de los cuales se afecta el crecimiento y la producción. Otros estudios han reportado resultados variables, por ejemplo, Urrestarazu 
y Mazuela (2005) al aplicar peróxido de potasio mediante el riego en melón, obtuvieron un incremento de $15 \%$ en rendimiento; mientras que en pepino no obtuvieron respuesta positiva a la oxifertirrigación. Por otra parte, Ehret et al. (2010) no obtuvieron diferencias en el rendimiento de pepino y pimiento utilizando un rango $\left[\mathrm{O}_{2}\right.$ ] desde 2 hasta $60 \mathrm{mg} / \mathrm{l}$.

En el cultivo de pimiento en perlita, Acuña et al. (2006) no encontraron efecto sobre parámetros de fertirrigación, altura de la planta, partición de biomasa y productividad, al aplicar concentraciones crecientes de $\mathrm{H}_{2} \mathrm{O}_{2}$ como oxigenante, desde $4,2 \mathrm{mg} \mathrm{O}_{2} / 1$ hasta $21,8 \mathrm{mg}$ $\mathrm{O}_{2} / 1$. Por el contrario, en pimiento, Marfa et al. (2005) y Urrestarazu y Mazuela (2005), obtuvieron incrementos en el rendimiento al aplicar oxifertirrigación química en la solución nutritiva. En cultivos de calabacín (Cucurbita pepo), soja (Glycine max) y algodón (Gossypium hirsutum) en un suelo arcilloso y con problemas de hipoxia, Bhattarai et al. (2004), obtuvieron un aumento en la biomasa total y en el rendimiento al aplicar $\mathrm{H}_{2} \mathrm{O}_{2}$ en fertirrigación.

La oxifertirrigación química, bajo el principio de "química verde" se basa en la utilización de sustancias químicas de fácil degradación, tales como el ácido peracético y el peróxido de hidrógeno, cuyos subproductos no producen contaminación ambiental (Carrasco y Urreztarazu, 2010). Una molécula de $\mathrm{H}_{2} \mathrm{O}_{2}$ se descompone en $\mathrm{H}_{2} \mathrm{O}$ y $\mathrm{O}_{2}$ (Tolvanen et al., 2010), incrementado la $\left[\mathrm{O}_{2}\right]$ en la rizosfera. Además, el $\mathrm{H}_{2} \mathrm{O}_{2}$ no tiene efectos carcinogénicos ni mutagénicos, posee alta eficiencia en aguas con alta concentración de materia orgánica y aplicado en fertirrigación ayuda al mantenimiento y limpieza del sistema de riego (Raffo, 2000).

Actualmente, Almería cuenta con un área de aproximadamente 4500 ha de cultivo en sustrato (Urreztarázu, 2004), la cual se ha mantenido estable en los últimos diez años. Inicialmente los sustratos más utilizados fueron perlita y lana de roca, y en menor cantidad la fibra de coco. Sin embargo, en los últimos años la fibra de coco se ha convertido en el principal sustrato utilizado en cultivos sin suelo. Entre las principales razones de dicho cambio está el aspecto ambiental. Tanto la perlita como la lana de roca, tiene una vida útil aproximada de dos años, al final de la cual representa un serio problema de residuos no biodegradables. Esta situación más la implementación de leyes ambientales rigurosas de la Unión Europea, obligaron a cerrar grandes fábricas de perlita y al cese de importaciones de lana de roca.
Por otra parte, las propiedades físicas de la fibra de coco, tales como una adecuada capacidad de retención de agua y capacidad de intercambio catiónico, facilitaban el manejo de la fertirrigación para los agricultores, teniendo una mayor capacidad buffer en comparación a los otros sustratos. Para los productores de cultivos sin suelo fue el medio de cultivo más similar a suelo desde el punto de vista de manejo. Actualmente, aunque no existen estudios oficiales, se estima que el $90 \%$ de los cultivos en sustrato, se realizan en fibra de coco, y el uso de otros sustratos va en clara decadencia.

El objetivo del presente trabajo fue evaluar el efecto del peróxido de hidrógeno $\left(\mathrm{H}_{2} \mathrm{O}_{2}\right)$ como fuente de oxígeno en la rizosfera, en tomate injertado (cv. Durinta/cv. Maxifor) y utilizando fibra de coco como sustrato.

\section{MATERIALES Y MÉTODOS}

El estudio se realizó en La Cañada de San Urbano, Almería, al sureste de España $\left(36^{\circ} 50^{\prime} \mathrm{N}\right.$ y $2^{\circ} 23^{\prime}$ W) en un ciclo de cultivo de tomate desde invierno (noviembre-2009) hasta inicio del verano (junio-2010).

Los promedios globales en el ciclo de cultivo, de temperaturas máximas, mínimas y medias, fueron $23,7,15,5$ y $19,5^{\circ} \mathrm{C}$, respectivamente. Para la humedad relativa, los promedios globales de máximas, mínimas y medias fueron de $83 \%, 45 \%$ y $65 \%$, respectivamente. El promedio global de radiación solar acumulada fue de $18,3 \mathrm{MJ} / \mathrm{m}^{2} / \mathrm{d}$.

Se cultivó tomate de ramo ( $c v$. Durinta) injertado (cv. Maxifort) en invernadero, y se empleó como sustrato la fibra de coco en sacos de $291(1,0 \mathrm{x}$ $0,18 \times 0,16 \mathrm{~m})$. Durinta es un cultivar de planta compacta y de buen vigor, con frutos calibre $\mathrm{M}$ muy uniformes en maduración para recolección en ramo; está recomendada para trasplantes tempranos de otoño-invierno, coincidente con la época de este estudio. Las características físicas, hidráulicas y las relaciones aire:agua del sustrato fueron determinadas previamente (Cuadro 1). Los sacos de cultivo fueron distribuidos en hileras simples espaciadas a $2 \mathrm{~m}$, sembrando tres plantas por saco cada $0,5 \mathrm{~m}$.

La densidad de siembra fue de una planta por $\mathrm{m}^{2}$ formada a dos tallos, los cuales fueron tutorados sobre alambre galvanizado sostenido por la estructura del invernadero. 
Cuadro 1. Análisis físico de la fibra de coco utilizada como sustrato en cultivo de tomate bajo invernadero. Almería, España. 2009-2010.

Table 1. Physical analysis of coconut fiber used as substrate in greenhouse hydroponic tomato. Almería, Spain. 2009-2010.

\begin{tabular}{|c|c|c|c|}
\hline \multirow[b]{2}{*}{ Parámetro } & \multicolumn{2}{|c|}{$\begin{array}{l}\text { Rangos } \\
\text { óptimos }^{1}\end{array}$} & \multirow{2}{*}{$\begin{array}{c}\text { Fibra } \\
\text { de coco }\end{array}$} \\
\hline & Máximo & Mínimo & \\
\hline Índice de grosor (\%) & & & 65 \\
\hline Densidad aparente $(\mathrm{g} / \mathrm{ml})$ & 0,15 & 0,15 & 0,07 \\
\hline Densidad real $(\mathrm{g} / \mathrm{ml})$ & 1.0 & 1,0 & 0,65 \\
\hline Porosidad total (\%) & - & $>85$ & 90 \\
\hline Fracción sólida (\%) & 15 & 15 & 10 \\
\hline Componentes totales & 100 & 100 & 100 \\
\hline \multicolumn{4}{|l|}{ Humedad volumétrica (\%) } \\
\hline $0 \mathrm{kPa}$ (saturado) & & & 90 \\
\hline $1 \mathrm{kPa}$ (capacidad de contenedor) & 55 & 70 & 61 \\
\hline $2,5 \mathrm{kPa}$ & & & 44 \\
\hline $5 \mathrm{kPa}$ & 30 & 40 & 38 \\
\hline $10 \mathrm{kPa}$ (punto de marchitez) & 25 & 30 & 32 \\
\hline \multicolumn{4}{|l|}{ Capacidad de aireación (\%) } \\
\hline $0 \mathrm{kPa}$ (saturado) & & & 0 \\
\hline $1 \mathrm{kPa}$ (capacidad de contenedor) & 30 & 15 & 29 \\
\hline $2,5 \mathrm{kPa}$ & 15 & 15 & 46 \\
\hline $5 \mathrm{kPa}$ & 55 & 45 & 52 \\
\hline $10 \mathrm{kPa}$ (punto de marchitez) & 60 & 55 & 58 \\
\hline \multicolumn{4}{|l|}{ Tipos de agua $(\%)$} \\
\hline Agua fácilmente disponible & 20 & 30 & 23 \\
\hline Agua de reserva & 5 & 10 & 6 \\
\hline Agua total disponible & 25 & 40 & 28 \\
\hline Agua difícilmente disponible & 30 & 30 & 32 \\
\hline
\end{tabular}

1 Rangos óptimos para sustratos según Ansorena (1994) / Optimal ranges for substrates according to Ansorena (1994).

Durante el ciclo del cultivo se realizaron labores de deshoja y poda de brotes laterales. Cuando en el cultivo se cuajó, aproximadamente el ramo número 12 , se realizó el despunte o poda del brote apical de crecimiento, con el objetivo de favorecer la distribución de asimilados hacia el llenado de frutos.

Para la nutrición mineral se utilizó una solución nutritiva estándar para cultivos comerciales de tomate con manejo convencional de la zona (Camacho, 2003), la cual fue distribuida en tres tanques: 1- nitrato de calcio y microelementos; 2- fosfato monopotásico, nitrato de potasio y sulfato de magnesio y 3-ácido nítrico. Se utilizó un sistema de fertirrigación abierto, donde el exceso de solución nutritiva aplicada en cada riego drenó a través de agujeros realizados en la parte inferior de los sacos de cultivo. De esta forma, la solución drenada no se recicló y fue liberada al ambiente. Para ello, se realizaron dos cortes o agujeros laterales en forma de $\mathrm{T}$ invertida en la parte inferior del saco de cultivo, lo más pegados al suelo para evitar acumulación de agua. Si había pendiente, se realizó un tercer agujero en el extremo inferior del saco.

El volumen y frecuencia de riegos se programó por tiempos y se ajustó de acuerdo a la $\mathrm{CE}$ en la solución drenada y al porcentaje de drenaje diario. La fertilización se inició con una CE de $0,75 \mathrm{dS} / \mathrm{m}$ durante la primera quincena, posteriormente se fue incrementando de acuerdo con las etapas fenológicas del cultivo hasta un máximo de $2,9 \mathrm{dS} / \mathrm{m}$ en la etapa de producción. Para el ajuste del pH se estableció un valor de consigna de 5,5 utilizando ácido nítrico (56\% y densidad de $167 \mathrm{~g} / \mathrm{l})$. El programa de fertirrigación fue controlado por ordenador, por medio de electroválvulas y venturi que controlaban la inyección de las soluciones fertilizantes en función de los valores de consigna de $\mathrm{CE}$ y $\mathrm{pH}$. El sistema de riego constó de dos sectores independientes, con tubería primaria de $25,5 \mathrm{~mm}$ y laterales de riego de $16 \mathrm{~mm}$, correspondiente con cada hilera de cultivo. Se utilizó un gotero por planta, autocompensado y antidrenante ( $3 \mathrm{l} / \mathrm{h}$ ), extendido con un microtubo y al extremo un gotero estaca que se fijó en el sustrato.

Se utilizaron dos tratamientos en fertirrigación: un control sin $\mathrm{H}_{2} \mathrm{O}_{2}\left(\mathrm{~T}_{0}\right)$ y otro con $\mathrm{H}_{2} \mathrm{O}_{2}\left(\mathrm{~T}_{1}\right)$, a una concentración de $100 \mathrm{mg} / \mathrm{l}$ utilizando un producto de uso comercial $\left(35 \% \mathrm{H}_{2} \mathrm{O}_{2}\right)$. El producto fue diluido en agua en una proporción volumétrica de 1:1, y posteriormente inyectado en forma continua en cada riego, por medio de una bomba dosificadora. De esta forma, se aportó una concentración constante de $\mathrm{O}_{2}$, asegurando su disponibilidad en la rizosfera. Para seleccionar la concentración de $\mathrm{H}_{2} \mathrm{O}_{2}$ en el $\mathrm{T}_{1}$, se condideró que existe una reducción sustancial de la $\left[\mathrm{O}_{2}\right]$ desde el tanque de aplicación o punto de inyección hasta la salida en el gotero, la cual incrementa aún más en el agua de drenaje después de atravesar el perfil del sustrato (Bonachela, comunicación personal, 2009).

Se utilizó un diseño experimental de bloques completos al azar con tres repeticiones, con hileras 
externas como cultivo de borde en cada bloque. El área experimental constó de un total de noventa plantas de tomate injertado, formadas a dos tallos. La unidad experimental (UE) estuvo formada por cinco sacos de cultivo sembrados con tres plantas cada uno, para un total de quince plantas de tomate por cada UE.

En cada tratamiento y repetición se midió la conductividad eléctrica, el pH y la $\left[\mathrm{O}_{2}\right]$ en solución nutritiva aplicada y en solución drenada. Para ello, dos veces por semana se recolectaron submuestras $i$ - de solución nutritiva en dos goteros seleccionados al azar y ii- de drenaje en una bandeja con dos sacos de cultivo. Además, se determinó el porcentaje de drenaje como el cociente entre el volumen de agua drenado y el volumen de agua aplicado. Para la medición de dichos parámetros de riego se utilizó un conductivímetro-potenciómetro (Crison Mod. CE-25) con precisión de $\pm 0,02 \mathrm{dS} / \mathrm{m}$ y $\pm 0,01$ para $\mathrm{pH}, \mathrm{y}$ un oxigenómetro (HACH HQ30d) con un rango de $0,1-$ $20 \mathrm{mg} / \mathrm{l}$ y una precisión de $\pm 0,2 \mathrm{mg} / \mathrm{l}$.

Desde la primer quincena hasta la quincena 10 , se midió la altura de la planta $(\mathrm{cm})$ y el diámetro del tallo $(\mathrm{mm})$ en cinco plantas por UE, cuyo valor promedio se consideró una repetición. La altura de la planta se midió con una cinta métrica desde la superficie del suelo hasta el brote terminal de la planta, mientras que el diámetro se midió en la base del tallo con un calibrador digital (CALIPER) con rango de 0-150 milímetros y una precisión de $\pm 0,01 \mathrm{~mm}$.

Durante el periodo de producción, para cada tratamiento y repetición, se evaluó el rendimiento comercial, no comercial y total, en las quince plantas por unidad experimental. El rendimiento comercial se clasificó por categorías de calidad de fruto (extra, I y II), de acuerdo al Reglamento de la Unión Europea (CE) No 717/2001 (Anon., 2001).

Además, en cada cosecha se evaluó la calidad externa (firmeza) e interna (grados Brix, $\mathrm{pH}$ y materia seca) del fruto. Para ello, se recolectaron al azar seis frutos por unidad experimental, cuyo valor promedio fue considerado como una repetición. Se seleccionaron frutos sanos, de tamaño uniforme y de un grado de madurez representativo de los estándares que se utilizaban a nivel comercial al momento de la cosecha.

El pH y grados Brix se midieron en el jugo extraído en los frutos macerados con un peachímetro (Crison Mod. CE-25) y un refractómetro (ATAGO-N1, con rango de $0-32 \%$ y precisión de $\pm 0,2 \%$ ). Para determinar la firmeza de fruto (pericarpo) y de pulpa (mesocarpo), se utilizó un penetrómetro (Bertuzzi FT327) con vástago de ocho $\mathrm{mm}$ y rango de $0-13$ $\mathrm{kg} / \mathrm{cm}^{2}$. El contenido de materia seca se determinó en estufa de convección forzada a $70{ }^{\circ} \mathrm{C}$ (modelo Mettler PM 4600) hasta peso constante.

Los datos experimentales obtenidos fueron sometidos a un análisis de varianza, verificando previamente los supuestos de normalidad y homocedasticidad. Cuando el estadístico $F$ del ANDEVA fue significativo $(\mathrm{P}<0,05)$, se realizó la comparación múltiple de medias según el procedimiento de diferencia mínima significativa (LSD) de Fisher $(\mathrm{P}<0,05)$. Todos los procedimientos estadísticos fueron realizados con el programa Statgraphics Centurión XV.

\section{RESULTADOS Y DISCUSIÓN}

\section{Efecto en el pH y CE de la solución nutritiva}

En general, no se observó efecto sobre el $\mathrm{pH}$ y $\mathrm{CE}$, al inyectar de forma continua $\mathrm{H}_{2} \mathrm{O}_{2}$ en el agua de riego. Los valores promedio de $\mathrm{pH}$ durante el ciclo de cultivo en ambos tratamientos, variaron en un rango de 5,0 hasta 6,3 (Figura 1), con un valor promedio global de 5,43 en el tratamiento $\mathrm{T}_{0}$ y de 5,84 en $\mathrm{T}_{1}$. No hubo diferencias estadísticamente significativas $(\mathrm{P}>0,05)$ entre tratamientos, en los valores de $\mathrm{pH}$ durante los periodos quincenales. Por tanto, la oxifertirrigación no tuvo efecto sobre el crecimiento y el rendimiento, debido a que el $\mathrm{pH}$ en solución nutritiva en gotero, en ambos tratamientos, estuvo dentro del rango óptimo para el cultivo de tomate. $\mathrm{Al}$ respecto, en estudios realizados, Dyśko et al. (2009) obtuvieron el máximo rendimiento en el cultivo de tomate a pH 5,5; mientras que Castellanos (2006) no obtuvo efectos negativos sobre la producción de tomate en fibra de coco entre tratamientos con valores promedio de $\mathrm{pH}$ de 5,02 y 4,82 .

En ambos tratamientos, la CE se inició con valores bajos (promedio de 0,76), incrementando progresivamente hasta 1,8 en la quincena 3 donde se mantuvo constante hasta la quincena 9 (Figura 1). Posteriormente, la CE se incrementó paulatinamente hasta la quincena 11 , donde mostró una tendencia a mantenerse estable en el tratamiento $\mathrm{T}_{1} \mathrm{y}$ una tendencia al incremento en $\mathrm{T}_{0}$. Consecuentemente, se presentaron diferencias significativas $(\mathrm{P}<0,05)$ entre tratamientos en la quincena 13 y 14 (Figura 1), alcanzando valores máximos al final del ciclo de $2,5 \mathrm{dS} / \mathrm{m}$ en $\mathrm{T}_{1}$ y $2,9 \mathrm{dS} / \mathrm{m}$ 


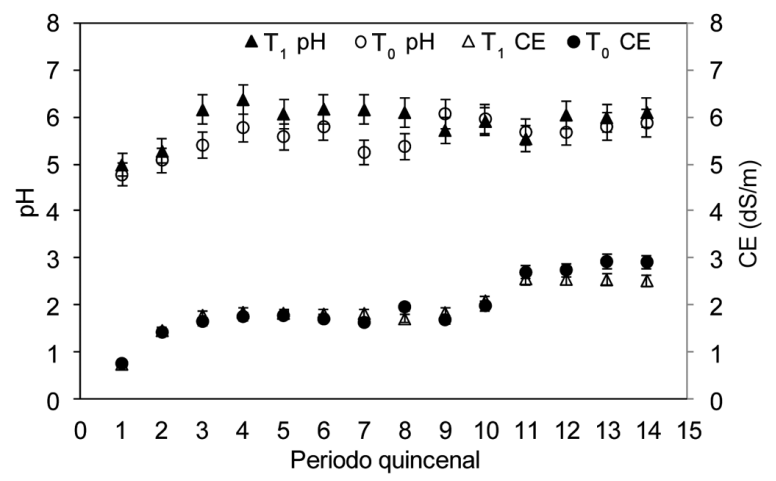

Figura 1. Valores promedio por quincena de $\mathrm{pH}$ y conductividad eléctrica de la solución nutritiva obtenida en gotero de los tratamientos con y sin oxifertirrigación, en un cultivo de tomate bajo invernadero. Almería, España. 2009-2010.

Las barras indican la desviación estándar de los promedios.

$\Delta \mathrm{T}_{1} \mathrm{pH}$ : $\mathrm{pH}$ en tratamiento con oxifertirrigación. $\bigcirc \mathrm{T}_{0} \mathrm{pH}$ : $\mathrm{pH}$ en tratamiento sin oxifertirrigación.

$\Delta \mathrm{T}_{1} \mathrm{CE}$ : conductividad eléctrica en tratamiento con oxifertirrigación.

- $\mathrm{T}_{0} \mathrm{CE}$ : conductividad eléctrica en tratamiento sin oxifertirrigación.

Figure 1. Fortnight average values of $\mathrm{pH}$ and electrical conductivity of the nutrient solution collected in dropper of treatments with and without oxifertigation, in greenhouse tomato crop. Almería, Spain. 2009-2010.

Bars indicate the standard deviation of averages.

$\Delta \mathrm{T}_{1} \mathrm{pH}: \mathrm{pH}$ in treatment with oxifertigation. $\bigcirc \mathrm{T}_{0} \mathrm{pH}: \mathrm{pH}$ in treatment without oxifertigation. $\Delta \mathrm{T}_{1} \mathrm{EC}$ : Electrical conductivity in treatment with oxifertigation.

- $\mathrm{T}_{0} \mathrm{CE}$ : Electrical conductivity in treatment without oxifertigation.

en $\mathrm{T}_{0}$. Estas diferencias no fueron un factor limitante para el crecimiento y la producción. Además, los valores promedio globales, fueron de $1,99 \mathrm{dS} / \mathrm{m}$ en $\mathrm{T}_{0}$ y $2,04 \mathrm{dS} / \mathrm{m}_{\text {en }} \mathrm{T}_{1}$. Al respecto, estudios realizados por Magán et al. (2008), demostraron que en el cultivo de tomate sin suelo el rendimiento se redujo a partir de valores de CE de 3,3 dS/m en solución nutritiva.

\section{Efectos sobre parámetros de drenaje}

No se presentaron diferencias significativas $(\mathrm{P}>0,05)$ entre tratamientos, en los valores promedio de
$\mathrm{pH}$ en drenaje durante el ciclo de cultivo, mientras los valores promedio globales fueron similares (alrededor de 6,5) en ambos tratamientos (Figura 2a).

Por otra parte, tampoco se observaron diferencias estadísticas $(\mathrm{P}>0,05)$ entre tratamientos, en los valores promedio de $\mathrm{CE}$ durante el ciclo de cultivo, mientras que los valores promedio globales fueron $2,45 \mathrm{dS} / \mathrm{m}$ en $\mathrm{T}_{0}$ y $2,38 \mathrm{dS} / \mathrm{m}$ en $\mathrm{T}_{1}$. La evolución de los valores de $\mathrm{CE}$ en las diferentes quincenas del ciclo de cultivo (Figura 2a) mostró un comportamiento similar al patrón de evolución de valores de CE en la solución en gotero (Figura 1). Los mayores valores de CE se presentaron desde la quincena 10 hasta el final del cultivo, debido a que este periodo coincidió con el inicio de la primavera, donde se registraron incrementos en la radiación solar y la temperatura y una reducción de la humedad ambiental, que consecuentemente incrementó la evapotranspiración del cultivo. Al comparar los valores promedio global de CE de la solución nutritiva en el gotero respecto a los valores de CE obtenidos en drenaje, se observaron diferencias de alrededor de $0,5 \mathrm{dS} / \mathrm{m}$, lo cual según Magán et al. (2008), desde el punto de vista práctico de manejo del cultivo, se considera aceptable.

Los valores de porcentaje de drenaje en ambos tratamientos mostraron un comportamiento muy irregular durante el ciclo de cultivo, variando desde $15 \%$ en la quincena 7 para $\mathrm{T}_{0}$ hasta $62 \%$ en la quincena 1 para $\mathrm{T}_{1}$ (Figura $2 \mathrm{~b}$ ). Los valores promedio estacional, fueron muy similares entre tratamientos, con $33 \%$ para $\mathrm{T}_{0}$ y $35 \%$ en $\mathrm{T}_{1}$. La mayor diferencia (15\%) entre tratamientos se presentó en la quincena 10. En general, los valores de drenaje obtenidos en las diferentes quincenas, se consideran normales para el manejo de riego en cultivos sin suelo en condiciones con aguas de regular calidad (Magán et al., 2008), lo cual sugiere que el manejo del riego en ambos tratamientos, estuvo dentro de los rangos normales de manejo convencional utilizado en cultivos comerciales de la región.

\section{Efecto en los parámetros de crecimiento}

No se observaron diferencias significativas $(\mathrm{P}>0,05)$ entre tratamientos en los valores de diámetro basal del tallo y altura de planta durante el ciclo de cultivo, mostrando una tendencia lineal al incremento (Figuras 3a y 3b). Esto sugiere que se realizó un buen manejo agronómico del cultivo respecto a prácticas de 

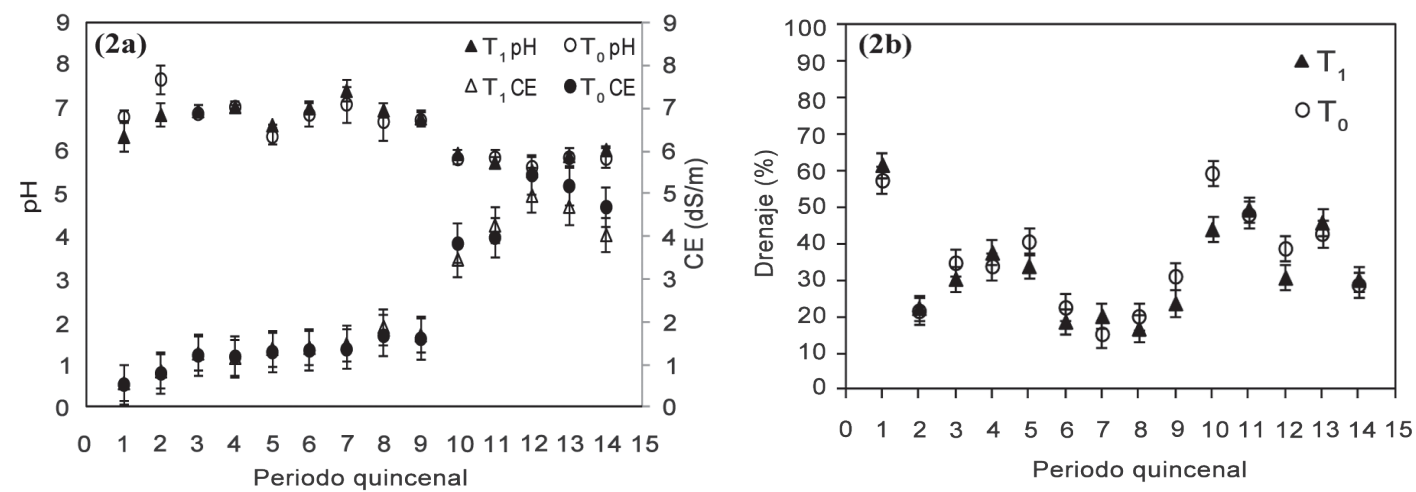

Figura 2. Valores promedio por quincena de $\mathrm{pH}$ y conductividad eléctrica en drenaje (2a) y porcentaje de drenaje (2b), en un cultivo de tomate en invernadero bajo tratamientos con y sin oxifertirrigación. Almería, España. 2009-2010. Las barras indican la desviación estándar de los promedios.

$\Delta \mathrm{T}_{1} \mathrm{pH}$ : $\mathrm{pH}$ en tratamiento con oxifertirrigación, $\bigcirc \mathrm{T}_{0} \mathrm{pH}$ : $\mathrm{pH}$ en tratamiento sin oxifertirrigación, $\Delta \mathrm{T}_{1} \mathrm{CE}$ : conductividad eléctrica en tratamiento con oxifertirrigación, $\bullet \mathrm{T}_{0} \mathrm{CE}$ : conductividad eléctrica en tratamiento sin oxifertirrigación, $\boldsymbol{\Delta}_{1}$ : Porcentaje de drenaje en tratamiento con oxifertirrigación, $\bigcirc \mathrm{T}_{0}$ : Porcentaje de drenaje en tratamiento sin oxifertirrigación.

Figure 2. Fortnight average values of $\mathrm{pH}$ and electrical conductivityof drainage (2a) and drainage percentage (2b), in greenhouse tomato crop under treatments with and without oxifertigation. Almería, Spain. 2009-2010. Bars indicate the standard deviation of averages.

$\Delta \mathrm{T}_{1} \mathrm{pH}: \mathrm{pH}$ in treatment with oxifertigation, $\bigcirc \mathrm{T}_{0} \mathrm{pH}: \mathrm{pH}$ in treatment without oxifertigation, $\Delta \mathrm{T}_{1} \mathrm{CE}$ : Electrical conductivity in treatment with oxifertigation, $\bullet \mathrm{T}_{0} \mathrm{CE}$ : Electrical conductivity in treatment without oxifertigation, $\boldsymbol{\Delta} \mathrm{T}_{1}$ : Percentage of drainage in treatment with oxifertigation, $\bigcirc \mathrm{T}_{0}$ : Percentage of drainage in treatment without oxifertigation
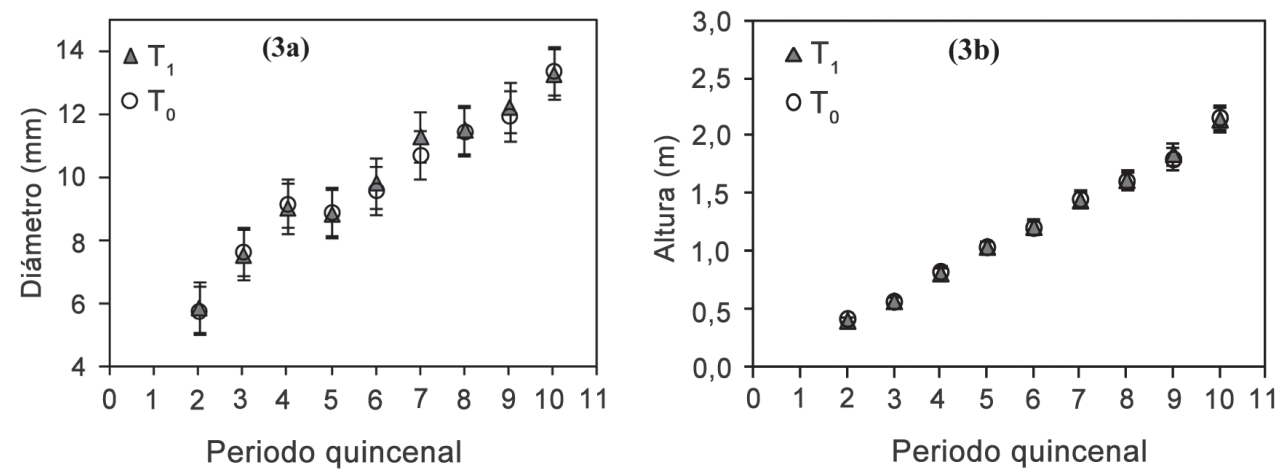

Figura 3. Valores promedio por quincena de diámetro basal del tallo (3a) y altura de la planta (3b), en un cultivo de tomate en invernadero bajo tratamientos con y sin oxifertirrigación. Almería, España. 2009-2010.

Las barras indican la desviación estándar de los promedios.

$\Delta \mathrm{T}_{1}$ : tratamiento con oxifertirrigación, $\bigcirc \mathrm{T}_{0}$ : tratamiento sin oxifertirrigación.

Figure 3. Fortnight average values of basal stem diameter (3a) and plant height (3b), in greenhouse tomato crop under treatments with and without oxifertigation. Almería, Spain. 2009-2010.

Bars indicate the standard deviation of averages.

$\mathbf{\Delta}_{1}$ : treatment with oxifertigation, $\bigcirc \mathrm{T}_{0}$ : treatment without oxifertigation. 
cultivo y fertirrigación, por lo cual no se presentaron condiciones limitantes de agua, $\mathrm{pH}$ y salinidad. En varios estudios realizados en tomate, se ha demostrado que a partir de una $\left[\mathrm{O}_{2}\right]$ de $3 \mathrm{mg} / \mathrm{l}$ no se afectó el crecimiento del cultivo (Gislerod y Adam, 1983; Zheng et al., 2007; Bonachela et al., 2010). En el presente estudio, los valores promedio globales de $\left[\mathrm{O}_{2}\right]$ en solución nutritiva, fueron $9,92 \mathrm{mg} / \mathrm{l}$ para $\mathrm{T}_{0} \mathrm{y}$ $12,1 \mathrm{mg} / \mathrm{l}$ en $\mathrm{T}_{1}$; lo cual sugiere que la disponibilidad de $\mathrm{O}_{2}$ no fue un factor limitante del crecimiento.

\section{Efecto en los parámetros de producción}

El tratamiento con oxifertirrigación química $\left(\mathrm{T}_{1}\right)$ no tuvo efecto sobre la producción de tomate comercial por categorías, comercial total y no comercial (Figura 4). Estos resultados son consistentes con los obtenidos por Bonachela et al. (2010), quienes no obtuvieron efecto sobre la producción al aplicar oxifertirrigación

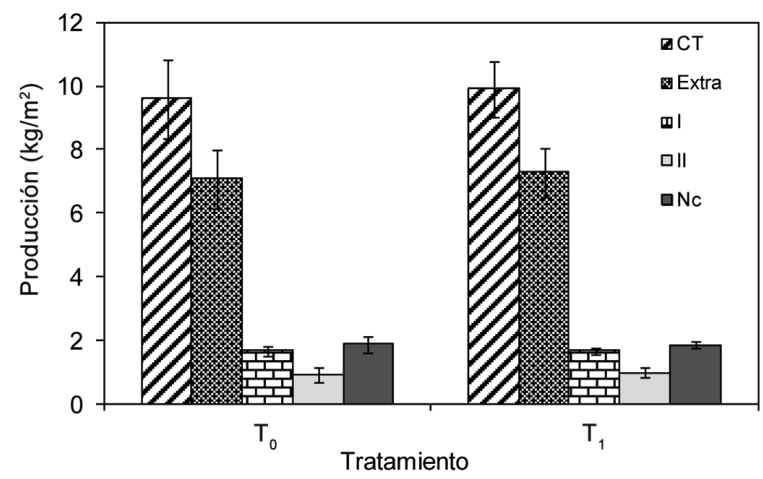

Figura 4. Producción de fruto comercial total por categorías y no comercial, en un cultivo de tomate en invernadero bajo tratamientos con y sin oxifertirrigación. Almería, España. 2009-2010.

Las barras indican la desviación estándar de los promedios.

CT: producción comercial total; Extra: producción de calidad extra; I: producción de primera calidad; II: producción de segunda calidad; Nc: producción no comercial.

Figure 4. Commercial fruit total production by category and noncommercial, in greenhouse tomato crop under treatments with and without oxifertigation. Almería, Spain. 2009-2010.

Bars indicate the standard deviation of averages.

CT: commercial total production; Extra: extra quality production; I: first quality production; II: second quality production; Nc: noncommercial production. a la solución nutritiva en el cultivo de tomate en lana de roca. La producción comercial obtenida en $T_{1}(9,6$ $\left.\mathrm{kg} / \mathrm{m}^{2}\right)$ y $\mathrm{T}_{0}\left(9,9 \mathrm{~kg} / \mathrm{m}^{2}\right)$, estuvo dentro del rango de rendimientos usuales de la región, reportados por Camacho (2003), para el cultivo de tomate en ramo bajo invernadero $\left(7,0-9,0 \mathrm{~kg} / \mathrm{m}^{2}\right)$.

\section{Efecto sobre la calidad de fruto}

El tratamiento con peróxido de hidrógeno no tuvo efecto estadísticamente significativo $(\mathrm{P}>0,05)$ sobre la firmeza de fruto, de pulpa, materia seca, grados Brix y $\mathrm{pH}$, durante las diferentes quincenas del ciclo de cultivo, mostrando pequeñas diferencias entre $T_{0}$ y $T_{1}$ en el valor promedio global al final del ciclo de cultivo (Cuadro 2). En estudios realizados por Castellanos (2006), no se encontraron diferencias significativas $(\mathrm{P}>0,05)$ en la firmeza de frutos, entre tratamientos con y sin aireación de la solución nutritiva, en tomate cultivado en fibra de coco. En otros estudios similares realizados en cultivo de tomate en sustrato con tratamiento de oxifertirrigación de la solución nutritiva, no se encontró efecto sobre los grados Brix y el pH del fruto (Bonachela et al., 2010).

\section{Concentración de oxígeno}

Una mayor $\left[\mathrm{O}_{2}\right]$ en la solución de riego y de drenaje en el tratamiento con peróxido de hidrógeno, no influyó en los parámetros de fertirrigación, tales como el $\mathrm{pH}$, la $\mathrm{CE}$ y el porcentaje de drenaje, ni en los parámetros de crecimiento definidos por el diámetro basal y la altura del cultivo de tomate. Esto debido a que en el tratamiento sin oxifertirrigación no hubo concentraciones limitantes de oxígeno, tanto en la solución nutritiva aplicada como en el drenaje. Además, la aplicación de $\mathrm{H}_{2} \mathrm{O}_{2}$ como oxigenante no tuvo efecto sobre el rendimiento y calidad del fruto de tomate caracterizado por la firmeza, contenido de materia seca, grados Brix y $\mathrm{pH}$.

Durante las quincenas 5, 7 y 13 se presentaron diferencias estadísticamente significativas $(\mathrm{P}<0,05)$ entre tratamientos, en los valores promedio de $\left[\mathrm{O}_{2}\right]$ en la solución de riego aplicada (Figura 5a); mientras que en la solución de drenaje se observaron diferencias estadísticas $(\mathrm{P}<0,05)$ en las quincenas 1 y 10 (Figura $5 b$ ). Los valores promedio de $\left[\mathrm{O}_{2}\right]$ en solución nutritiva para todo el ciclo completo fueron $9,92 \mathrm{mg} / 1$ para $\mathrm{T}_{0}$ y $12,1 \mathrm{mg} / \mathrm{l}$ en $\mathrm{T}_{1}$; mientras que en 
Cuadro 2. Valores promedio \pm desviación estándar, por semana de cosecha y ciclo de cultivo, para las variables de firmeza de fruto y de pulpa, y materia seca, grados Brix y pH de frutos de tomate en invernadero bajo tratamientos con y sin oxifertirrigación. Almería, España. 2009-2010.

Table 2. Mean values \pm standard deviation per week of harvest and crop cycle for the variables of fruit firmness and pulp, and dry matter, brix degrees and pH of tomato fruits under treatment with and without oxifertigation. Almería, Spain. 2009-2010.

\begin{tabular}{|c|c|c|c|c|c|c|c|c|c|c|c|c|c|c|c|c|}
\hline \multirow{3}{*}{$\begin{array}{c}\text { Semana } \\
1\end{array}$} & \multirow{3}{*}{$\begin{array}{l}\mathbf{T r}^{1} \\
\mathrm{~T}_{1}\end{array}$} & \multicolumn{6}{|c|}{ Firmeza $\left(\mathrm{kg} / \mathrm{cm}^{2}\right)$} & \multirow{2}{*}{\multicolumn{3}{|c|}{$\begin{array}{c}\text { Materia seca } \\
(\%)\end{array}$}} & \multirow{2}{*}{\multicolumn{3}{|c|}{ Grados Brix }} & \multirow{2}{*}{\multicolumn{3}{|c|}{ pH }} \\
\hline & & \multicolumn{3}{|c|}{ Fruto } & \multicolumn{3}{|c|}{ Pulpa } & & & & & & & & & \\
\hline & & 3,41 & \pm & 0,23 & 3,26 & \pm & 0,02 & 5,29 & \pm & 0,12 & 6,64 & \pm & 0,44 & 4,28 & \pm & 0,12 \\
\hline & $\mathrm{T}_{0}$ & 3,74 & \pm & 0,24 & 3,27 & \pm & 0,29 & 5,72 & \pm & 0,21 & 7,29 & \pm & 0,42 & 4,04 & \pm & 0,44 \\
\hline 2 & $\mathrm{~T}_{1}$ & 4,53 & \pm & 0,47 & 2,53 & \pm & 0,38 & 5,85 & \pm & 0,41 & 7,02 & \pm & 0,30 & 3,77 & \pm & 0,06 \\
\hline & $\mathrm{T}_{0}$ & 4,50 & \pm & 0,60 & 3,08 & \pm & 0,65 & 5,72 & \pm & 0,87 & 6,97 & \pm & 0,33 & 3,80 & \pm & 0,10 \\
\hline 3 & $\mathrm{~T}_{1}$ & 3,87 & \pm & 0,26 & 2,27 & \pm & 0,29 & 5,51 & \pm & 0,33 & 7,12 & \pm & 0,36 & 4,01 & \pm & 0,04 \\
\hline & $\mathrm{T}_{0}$ & 3,48 & \pm & 0,57 & 2,41 & \pm & 0,30 & 5,64 & \pm & 0,45 & 7,07 & \pm & 0,28 & 3,89 & \pm & 0,15 \\
\hline 4 & $\mathrm{~T}_{1}$ & 3,86 & \pm & 0,59 & 2,41 & \pm & 0,49 & 4,86 & \pm & 0,63 & 6,96 & \pm & 0,04 & 3,96 & \pm & 0,16 \\
\hline & $\mathrm{T}_{0}$ & 3,76 & \pm & 0,32 & 2,02 & \pm & 0,21 & 5,84 & \pm & 0,65 & 6,93 & \pm & 0,03 & 3,86 & \pm & 0,12 \\
\hline 5 & $\mathrm{~T}_{1}$ & 4,69 & \pm & 0,63 & 3,13 & \pm & 0,32 & 5,72 & \pm & 0,15 & 6,97 & \pm & 0,15 & 3,77 & \pm & 0,14 \\
\hline & $\mathrm{T}_{0}$ & 5,14 & \pm & 0,78 & 3,23 & \pm & 0,42 & 5,92 & \pm & 0,52 & 6,72 & \pm & 0,07 & 3,88 & \pm & 0,07 \\
\hline 6 & $\mathrm{~T}_{1}$ & 4,42 & \pm & 0,23 & 3,02 & \pm & 0,20 & 5,31 & \pm & 0,33 & 6,69 & \pm & 0,24 & 4,14 & \pm & 0,07 \\
\hline & $\mathrm{T}_{0}$ & 4,07 & \pm & 0,86 & 2,83 & \pm & 0,30 & 5,39 & \pm & 0,45 & 7,02 & \pm & 0,08 & 4,22 & \pm & 0,07 \\
\hline 7 & $\mathrm{~T}_{1}$ & 4,30 & \pm & 0,26 & 2,91 & \pm & 0,28 & 5,43 & \pm & 0,14 & 6,59 & \pm & 0,17 & 4,14 & \pm & 0,03 \\
\hline & $\mathrm{T}_{0}$ & 4,21 & \pm & 0,21 & 3,34 & \pm & 0,11 & 5,42 & \pm & 0,24 & 6,73 & \pm & 0,27 & 4,24 & \pm & 0,04 \\
\hline 8 & $\mathrm{~T}_{1}$ & 3,37 & \pm & 0,42 & 1,99 & \pm & 0,14 & 5,24 & \pm & 0,63 & 5,81 & \pm & 1,22 & 4,27 & \pm & 0,05 \\
\hline & $\mathrm{T}_{0}$ & 3,64 & \pm & 0,16 & 1,88 & \pm & 0,22 & 5,60 & \pm & 0,41 & 6,80 & \pm & 0,10 & 4,27 & \pm & 0,05 \\
\hline Por ciclo & $\mathrm{T}_{1}$ & 3,99 & \pm & 0,18 & 2,69 & \pm & 0,06 & 5,40 & \pm & 0,24 & 6,73 & \pm & 0,27 & 4,04 & \pm & 0,05 \\
\hline & $\mathrm{T}_{0}$ & 4,13 & \pm & 0,19 & 2,76 & \pm & 0,09 & 5,66 & \pm & 0,28 & 6,94 & \pm & 0,01 & 4,03 & \pm & 0,06 \\
\hline
\end{tabular}

${ }^{1} \mathrm{Tr}$ : tratamientos, $\mathrm{T}_{1}$ : tratamiento con oxifertirrigación y $\mathrm{T}_{0}$ : tratamiento sin oxifertirrigación / Tr: treatment, $\mathrm{T}_{1}$ : treatment with oxifertigation, $\mathrm{T}_{0}$ : treatment without oxifertigation.

solución drenada las $\left[\mathrm{O}_{2}\right]$ fueron 8,75 y $9,22 \mathrm{mg} / \mathrm{l}$, para $\mathrm{T}_{0} \mathrm{y} \mathrm{T}_{1}$, respectivamente.

En diversos estudios, se ha encontrado una alta variabilidad en la respuesta de los cultivos a la aplicación de tratamientos de oxigenación, con valores umbrales de $\left[\mathrm{O}_{2}\right]$ que varían desde $2 \mathrm{mg} / \mathrm{l}$ hasta $7 \mathrm{mg} / \mathrm{l}$ (Gislerod y Adam, 1983; Gislerod y Kempton, 1983; Zeroni et al., 1983; Acuña et al., 2008; Bonachela et al., 2010). Por otra parte, Bonachela et al., 2005, concluyeron que los valores de $\left[\mathrm{O}_{2}\right]$ en la solución del sustrato, durante la mayor parte del ciclo de cultivo de tomate, estuvieron por encima de los umbrales críticos determinados en dichos estudios. Lo anterior sugiere que los umbrales de $\left[\mathrm{O}_{2}\right]$ no están claramente definidos y que dependen del cultivo y de una serie de factores condicionantes. Aunque en algunas investigaciones se ha obtenido una respuesta positiva en rendimiento, calidad y crecimiento a la aplicación de oxígeno (Marfa y Gurí, 1999; Urrestarazu y Mazuela, 2005), no está claro si dichos estudios fueron realizados bajo alguna condición limitante de $\mathrm{O}_{2}$. En otros estudios, Battahari et al. (2004, 2006) y Battahari y Midmore (2009) al aportar oxígeno al agua en riego localizado en un suelo arcilloso con limitantes de oxígeno encontraron mayor productividad en varios cultivos, entre ellos el tomate.

Las causas más comunes que podrían inducir a una condición de hipoxia son: $i$ - un mal manejo o un exceso de riego (Drew, 1997; Bonachela et al., 2005), ii- malas características físicas del sustrato, iii- aguas de riego con baja $\left[\mathrm{O}_{2}\right]$, iv- altas temperaturas del sustrato en época seca (Bonachela, et al., 2005) y $v$ - una combinación de algunas o todas las anteriores causas. En este estudio, el agua presentó una adecuada $\left[\mathrm{O}_{2}\right]$, mientras que el sustrato presentó una buena capacidad 

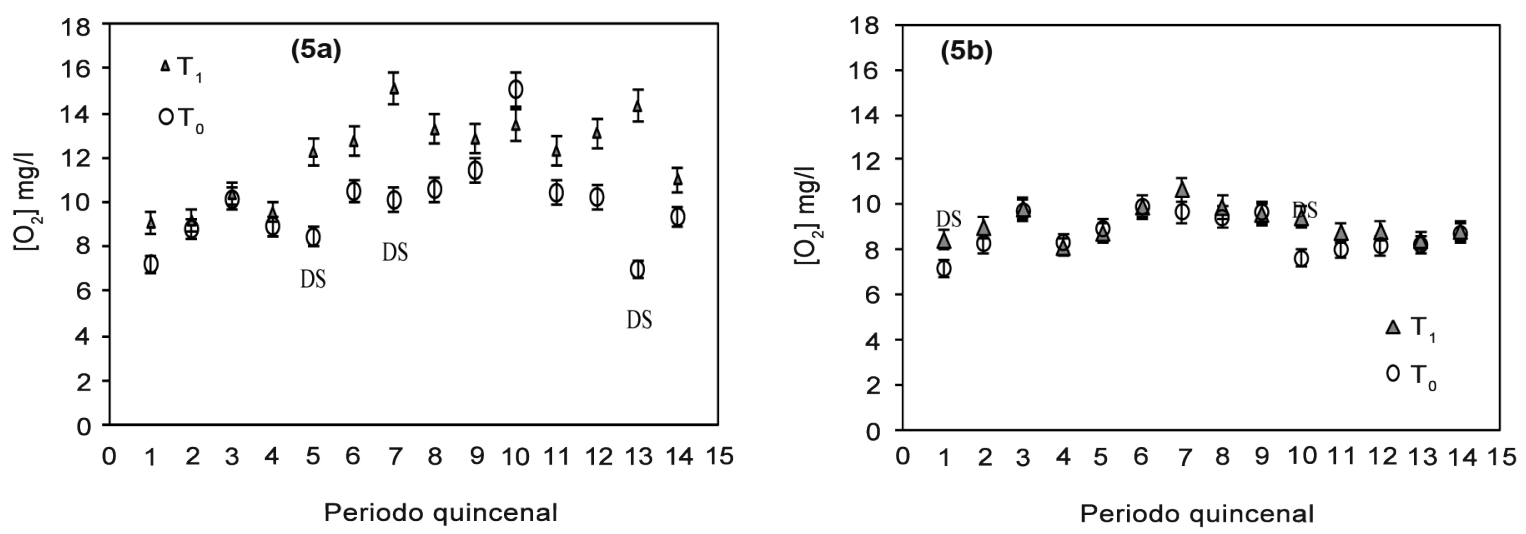

Figura 5. Valores promedio por quincena de concentración de oxígeno en solución nutritiva (5a) y en drenaje (5b), en un cultivo de tomate en invernadero bajo tratamientos con y sin oxifertirrigación. Almería, España. 2009-2010.

Las barras y DS indican la desviación estándar de los promedios y diferencias significativas entre tratamientos según prueba LSD $(\mathrm{P}<0,05)$, respectivamente.

$\Delta \mathrm{T}_{1}$ : tratamiento con oxifertirrigación, $\bigcirc \mathrm{T}_{0}$ : tratamiento sin oxifertirrigación.

Figure 5. Fortnight average values of oxygen concentration in nutrient solution (5a) and drainage (5b), in greenhouse tomato crop under treatments with and without oxifertigation. Almería, Spain. 2009-2010.

Bars and DS indicate the standard deviation of averages and significant differences between treatments according to LSD test $(\mathrm{P}<0,05)$, respectively.

$\Delta \mathrm{T}_{1}$ : treatment with oxifertigation, $\bigcirc \mathrm{T}_{0}$ : treatment without oxifertigation.

de aireación a capacidad de contenedor, de acuerdo a los rangos óptimos establecidos (Cuadro 1).

A pesar de las diferencias en la $\left[\mathrm{O}_{2}\right]$ entre $\mathrm{T}_{0}$ y $\mathrm{T}_{1}$, en ambos tratamientos las $\left[\mathrm{O}_{2}\right]$ en solución nutritiva de gotero y en drenaje, durante todo el ciclo de cultivo, fueron superiores a los valores umbrales reportados en la literatura. En el tratamiento sin oxifertirrigación $\left(\mathrm{T}_{0}\right)$ la $\left[\mathrm{O}_{2}\right]$ en la solución nutritiva de gotero fue $9,92 \mathrm{mg} / \mathrm{l}$, lo cual demuestra que no hubo condiciones limitantes de oxígeno en el agua de riego. En Almería, Bonachela et al. (2007) al evaluar la $\left[\mathrm{O}_{2}\right]$ en el agua de diferentes reservorios de zonas representativas de la región, encontraron que los valores promedio diarios de $\left[\mathrm{O}_{2}\right]$ variaron desde 11,3 a $15,7 \mathrm{mg} / \mathrm{l}$. Esto sugiere que probablemente la $\left[\mathrm{O}_{2}\right]$ en las aguas de riego de la zona, no representaron un factor limitante, por lo tanto, habría que prestar más atención a otros factores como probables causas de hipoxia.

Algunos investigadores han propuesto que una forma más objetiva para medir la $\left[\mathrm{O}_{2}\right]$ en la rizosfera, es midiendo directamente en la solución extraída del medio de cultivo. Al respecto, Ehret et al. (2010) demostraron que hay una reducción de hasta un 67\% durante el recorrido desde el tanque de aplicación a la salida del gotero. Por su parte, Bonachela et al. (2010) encontraron que en tratamientos con oxifertirrigación en tomate, la $\left[\mathrm{O}_{2}\right]$ disminuyó un $65 \%$ durante su recorrido desde el punto de aplicación $(14,6 \mathrm{mg} / \mathrm{l})$ hasta la solución extraída directamente del sustrato $(5,1 \mathrm{mg} / \mathrm{l})$. En otro estudio realizado en el cultivo de sandía utilizando como sustrato perlita, se demostró que la $\left[\mathrm{O}_{2}\right]$ medida directamente en la solución extraída del sustrato de un tratamiento con oxigenación fue similar a la del tratamiento sin oxigenación; a pesar de presentar diferencias estadísticas $(\mathrm{P}<0,05)$ en la $\left[\mathrm{O}_{2}\right]$ de la solución obtenida en goteros (Bonachela et al., 2005). Dichos autores señalan que solo de un 8 a 12\% de la solución del medio de cultivo se renueva en cada evento de riego, lo cual puede agravarse si se aplica una solución baja en $\left[\mathrm{O}_{2}\right]$. La difusión de $\left[\mathrm{O}_{2}\right]$ del espacio aéreo a la fase líquida de la solución de la rizosfera es lenta, sobre todo cuando aumenta la temperatura del sustrato y la tasa respiratoria se incrementa.

Para futuras investigaciones, antes de aplicar tratamientos de oxigenación, se deben evaluar las causas potenciales que podrían conducir a una condición de hipoxia, y considerar las siguientes recomendaciones: identificar si existen factores potenciales que pueden 
limitar una adecuada oxigenación en la rizosfera; establecer la relación causa-efecto y determinar si ¿es posible corregir la deficiencia de oxígeno mejorando el manejo del factor limitante, por ejemplo riego? o ¿es imprescindible corregir la hipoxia aplicando algún método de oxigenación? Para ello, se debe evaluar el sistema de cultivo a utilizar, las características físicas del medio de cultivo, la época climática, aspectos de manejo del riego y calidad del agua, entre otros. Además, se deben evaluar las características físicas del sustrato según la edad de uso y determinar si el sustrato ya cumplió su vida útil y si es necesario renovarlo. Por otra parte, se debe cuestionar si la baja $\left[\mathrm{O}_{2}\right]$ es una consecuencia de características físicas limitantes del sustrato, del clima cálido, de un mal manejo del riego o resultado de una combinación de los factores anteriores.

Una vez identificado el problema se debe reproducir la/s condición/es limitante/s en el experimento, aplicando tratamientos con oxigenación, sin oxigenación y un testigo en condiciones óptimas de aireación del sustrato y buena calidad de agua. Además, se deben establecer las etapas críticas, según la fenología del cultivo, en las cuales es probable que se presente hipoxia debido a factores limitantes previamente identificados. $\mathrm{La}\left[\mathrm{O}_{2}\right]$ disminuye durante las horas más cálidas del día (Gislerop y Adams, 1983; Urrestarazu et al., 2005; Bonachela et al., 2010), por lo cual deben considerarse las condiciones climáticas. $\mathrm{Al}$ respecto, Bonachela et al. (2005), demostraron que las condiciones limitantes de $\left[\mathrm{O}_{2}\right]$ ocurren en ciertos periodos críticos del ciclo de cultivo y se agudizan en épocas cálidas, siendo las etapas más críticas durante el cuajado y crecimiento del fruto, y en el periodo de elevado desarrollo radicular. En condiciones cálidas hay una mayor demanda de oxígeno disuelto a nivel de rizosfera, coincidiendo con una alta actividad metabólica y alta absorción de agua y minerales. Por esta razón, el enriquecimiento de oxígeno no parece necesario durante todo el ciclo de cultivo, excepto en etapas críticas donde se presente alguna condición limitante. Por otra parte, se debe considerar la sensibilidad de los diferentes cultivos a condiciones de baja $\left[\mathrm{O}_{2}\right]$ en la rizosfera, con el objetivo de establecer valores umbrales para cultivos específicos.

La oxifertirrigación se justifica en aquellos casos en que los niveles de oxígeno en la rizosfera son lo suficientemente bajos para afectar el crecimiento y la productividad del cultivo. Esto requiere del conocimiento, experiencia y seguimiento de los probables factores que inducen a hipoxia, la identificación de los periodos críticos según el cultivo y la temperatura según la época y hora del día.

\section{LITERATURA CITADA}

Abad, M., P. Noguera, y C. Carrión. 2004. Los sustratos en los cultivos sin suelo. En: M. Urrestarazu, editor, Tratado de cultivo sin suelo. $3^{\text {a }}$ edición. Ed. Mundi Prensa, Almería, ESP. p. 113-158.

Abad, M., P. Noguera, V. Noguera, A. Roig, J. Cegarra, y C. Paredes. 1997. Reciclado de residuos orgánicos y su aprovechamiento como sustratos de cultivo. Acta Hortic. 19:92-109.

Acuña, R., S. Bonachela, y J. Magán. 2006. Respuesta de un cultivo de pimiento en sustrato de perlita a la mejora de la oxigenación del medio radicular. Acta Hortic. 46:91-95.

Acuña, G., S. Bonachela, and J. Magán. 2008. Oxyfertigation of a greenhouse melon crop grown in rockwool slabs in a mediterranean area. Acta Hortic. ISHS 779:447-454.

Alcayde, G. 2009. Evaluación de la respuesta productiva y cualitativa de un cultivo de melón cv. Vulcano, cultivado en distintos sustratos de lana de roca y baja distintas construcciones de oxígeno disuelto en el agua de riego. Tesis de Ingeniero Agrónomo, Universidad de Almería, ESP.

Anón. 2001. Reglamento CE N ${ }^{\circ}$ 717/2001 de la Comisión, del 10 de abril de 2001, por el que se establecen las normas de comercialización aplicables a los tomates. En Diario Oficial de las Comunidades Europeas L 100:11. UE.

Ansorena, M. 1994. Sustratos, propiedades y caracterización. Editorial Mundi-Prensa, Madrid, ESP.

Barret-Lennard, E. 2003. The interaction between water logging and salinity in higher plants: causes, consequences and implications. Plant Soil 253:35-54.

Bhattarai, S., S. Huber, and D. Midmore. 2004. Aerated subsurface irrigation water gives growth and yield benefits to zucchini, vegetable soybean and cotton in heavy clay soils. Ann. Appl. Biol. 144:285-298.

Bhattarai, S., and D. Midmore. 2009. Oxygation enhances growth, gas exchange and salt tolerance of vegetable soybean and cotton in a saline vertisol. JIPB 51:675-688.

Bhattarai, S., L. Pendergast, and D. Midmore. 2006. Root aeration improves yield and water use efficiency of 
tomato in heavy clay and saline soils. Sci. Hortic. 108:278-288.

Bhattarai, S., N. Su, and D. Midmore. 2005. Oxygation unlocks yield potentials of crops in oxygen-limited soil environments. Adv. Agron. 88:313-377.

Bonachela, S., R. Acuña, and J. Casas. 2007. Environmental factors and management practices controlling oxygen dynamics in agricultural irrigation ponds in a semiarid mediterranean region: Implications for pond agricultural functions. Water Res. 41:1225-1234.

Bonachela, S., J. Quesada, R. Acuña, J. Magán, and O. Marfà. 2010. Oxyfertigation of a greenhouse tomato crop grown on rockwool slabs and irrigated with treated wastewater: Oxygen content dynamics and crop response. Agric. Water Manag. 97:433-438.

Bonachela, S., J. Vargas, and R. Acuña. 2005. Effect of increasing the dissolved oxygen in the nutrient solution to above-saturation levels in a greenhouse watermelon crop grown in perlite bags in a mediterranean area. Acta Hort. (ISHS) 697:25-32.

Camacho, F. 2003. Técnicas en producción de cultivos protegidos. Tomo 2. Ediciones agrotécnicas S.L. Cajamar, ESP.

Carrasco, G., and M. Urrestarazu. 2010. Green chemistry in protected horticulture: The use of peroxyacetic acid as a sustainable strategy. Int. J. Mol. Sci. 11:1999-2009.

Castellanos, D. 2006. Aireación radical a bajo costo en sistemas de cultivo sin suelo con diferentes sustratos. Tesis doctoral, Universidad de Almería, ESP.

Chérif, M., Y. Tirilly, and R. Bélanger. 1997. Effect of oxygen concentration on plant growth, lipidperoxidation, and receptivity of tomato roots to pythium $\mathrm{F}$ under hydroponic conditions. Eur. J. Plant Pathol. 103:255-264.

Devaux, C., P. Baldet, J. Joubès, M. Dieuaide-Noubhani, D. Just, and C. Chevalier. 2003. Physiological, biochemical and molecular analysis of sugar-starvation responses in tomato roots. J. Exp. Bot. 54:1143-1151.

Dyśko, J., W. Kowalczyk, and S. Kaniszewski. 2009. The influence of $\mathrm{pH}$ of nutrient solution on yield and nutritional status of tomato plants grown in soilless culture system. Veg. Crop. Res. Bull. 70:59-69.

Drew, M. 1997. Oxygen deficiency and root metabolism: Injury and acclimation under hypoxia and anoxia. Annu. Rev. Plant Physiol. Plant Mol. Biol. 48:223-250

Ehret, D., D. Edwards, T. Helmer, W. Lin, G. Jones, and M. Dorais. 2010. Effects of oxygen-enriched nutrient solution on greenhouse cucumber and pepper production. Sci. Hort. 125:602-607.
García, M., M. Guzmán, M. Urrestarazu, M. Salas, y I. Escobar. 1997. Evaluación de diferentes parámetros en cultivo de perlita para distintas especies hortícolas en invernadero. Acta Hortic. 18:519-525.

Gislerod, H., and P. Adams. 1983. Diurnal variations in the oxygen content and acid requirement of recirculating nutrient solutions and in the uptake of water and potassium by cucumber and tomato plants. Sci. Hort. 21:311-321.

Gislerod, H., and R. Kempton. 1983. The oxygen content of flowing nutrient solutions used for cucumber and tomato culture. Sci. Hort. 20:23-33.

Goto, E., A.J. Both, L.D. Albright, R.W. Langhans, and A.R. Leed. 1996. Effect of dissolved oxygen concentration on lettuce growth in floating hydroponics. Acta Hortic. 440:205-210.

Horchani, F., A. Aloui, R. Brouquisse, and S. Aschi-Smiti. 2008a. Physiological responses of tomato plants (Solanum lycopersicum) as affected by root hypoxia. J. Agron. Crop Sci. 194:297-303.

Horchani, F., P. Gallusci, P. Baldet, C. Cabasson, M. Maucourt, D. Rolin, S. Aschi-Smiti, and P. Raymond. 2008b. Prolonged root hypoxia induces ammonium accumulation and decreases the nutritional quality of tomato fruits. J. Plant Physiol. 165:1352-1359.

Horchani, F., H. Khayati, P. Raymond, R. Brouquisse, and S. Aschi-Smiti. 2009. Contrasted effects of prolonged root hypoxia on tomato root and fruit (Solanum lycopersicum) metabolism. J. Agron. Crop Sci. 195:313-318.

Irving, L., Y. Sheng, D. Woolley, and C. Matthew. 2007. Physiological effects of waterlogging on two lucerne varieties grown under glasshouse conditions. J. Agron. Crop Sci. 193:345-356.

Magán, J., M. Gallardo, R. Thompson, and P. Lorenzo. 2008. Effects of salinity on fruit yield and quality of tomato grown in soilless culture in greenhouses in mediterranean climatic conditions. Agric. Water Manag. 95:1041-1055.

Marfa, O., R. Cáceres, and S. Gurí. 2005. Oxyfertigation: a new technique for soilless culture under Mediterranean conditions. Acta Hort. 697:65-72.

Marfa, O., y S. Gurí. 1999. Física de sustratos y oxigenación del medio radicular. En: cultivo sin suelo II: Curso superior de especialización. Coord. por M. Fernández; Cuadrado, I. IFAPA y Caja Rural de Almería, Almería, ESP. p. 93-106.

Pezeshki, S. 2001. Wetland plant responses to soil flooding. Environ. Exp. Bot. 46:299-312. 
Pivot, T., A. Reist, J. Gilliost, and J. Ryser. 1998. Water quality, climat environment and mineral of tomato (Solanum lycopersicum L.) in closed soilless cropping system. Acta Hortic. 458:207-214.

Raffo, L. 2000. Empleo de compuestos equilibrados de ácido peracético y peróxido de hidrógeno como desinfectantes de alto nivel y esterilizantes líquidos. Lehigh University - USA. Senior Biochemistry Investigator-AOAC International, USA.

Stiepniewski, W., and Y.G. Przywara. 1992. The influence of soil oxygen availability on yield and nutrient uptake (N, P, K, Ca, Mg, Na) by winter rye (Secale cereale). Plant Soil 143:267-274.

Tolvanen, P., P. Mäki-Arvela, A. Sorokin, T. Salmi, and D. Murzin. 2010. Kinetics of starch oxidation using hydrogen peroxide as an environmentally friendly oxidant and an iron complex as a catalyst. Chem. Eng. J. 154(1-3):52-59.

Urrestarazu, M. 2004. Bases y sistemas de los cultivos sin suelo. En: M. Urrestarazu, editor, Tratado internacional de cultivo sin suelo. Ed. Mundi Prensa, Almeria, ESP. p. 3-48.
Urrestarazu, M., and P. Mazuela. 2005. Effect of slowrelease oxygen supply by fertigation on horticultural crops under soilless culture. Sci. Hort. 106:484-490.

Urrestarazu, M., P. Mazuela, A. Boukhalfa, A. Arán, and M. Salas. 2005. Oxygen content and its diurnal variation in a new recirculating water soilless culture for horticultural crops. HortSci. 40:1730-1731.

Vartapetian, B., and Y. Jackson. 1997. Plant adaptations to anaerobic stress. Ann. Bot. 79:3-20.

Zeroni, M., J. Gale, and J. Ben-Asher. 1983. Root aeration in a deep hydroponic system and its effect on growth and yield of tomato. Sci. Hort. 19:213-220.

Zheng, Y., L. Wang, and M. Dixon. 2007. An upper limit for elevated root zone dissolved oxygen concentration for tomato. Sci. Hort. 113:162-165.

Zhi, K., W.H. Hu, D.K. Dong, Y.H. Zhou, and J.Q. Yu. 2007. Low $\mathrm{O}_{2}$ supply is involved in the poor growth in rootrestricted plants of tomato (Solanum lycopersicum). Environ. Exp. Bot. 61:181-189. 
